AperTO - Archivio Istituzionale Open Access dell'Università di Torino

HPLC-MS method for the simultaneous quantification of the antileukemia drugs imatinib, dasatinib and nilotinib in human peripheral blood mononuclear cell (PBMC).

This is a pre print version of the following article:

Original Citation:

Availability:

This version is available http://hdl.handle.net/2318/111970

since 2021-04-26T12:04:30Z

Published version:

DOI:10.1016/j.jpba.2011.10.003

Terms of use:

Open Access

Anyone can freely access the full text of works made available as "Open Access". Works made available under a Creative Commons license can be used according to the terms and conditions of said license. Use of all other works requires consent of the right holder (author or publisher) if not exempted from copyright protection by the applicable law. 


\section{HPLC-MS method for the simultaneous quantification of the antileukemia drugs imatinib, dasatinib and nilotinib in human peripheral blood mononuclear cell (PBMC).}

Antonio D’Avolio* (BSc, MSc, SM)

Marco Simiele* (BSc, MSc)

Silvia De Francia ${ }^{\#}$ (BSc, MSc, PhD)

Alessandra Ariaudo" (BSc, MSc)

Lorena Baietto* (BSc, MSc)

Jessica Cusato* (BSc, MSc)

Carmen Fava" ${ }^{\#}$ MD)

Giuseppe Saglio" (MD)

Francesco Di Carlo" (MD)

Giovanni Di Perri* (MD, DTM \& H, PhD)

*Laboratory of Clinical Pharmacology and Pharmacogenetics**. Department of Infectious Diseases, University of Torino, Amedeo di Savoia Hospital, Turin, Italy

\#Department of Biological and Clinical Sciences, University of Torino, S. Luigi Gonzaga Hospital, Regione Gonzole 10, 10043 Orbassano (Turin), Italy.

**EN UNI ISO 9001:2008 Certificate Laboratory; Certificate No. IT-64386;

www.tdm-torino.org

Corresponding author:

Antonio D'Avolio, (BSc, MSc, SM)

Tel. +39.011.4393979, Fax: +39.011.4393882

e-mail: antonio.davolio@unito.it

Keywords: imatinib; dasatinib; nilotinib; HPLC-MS; intracellular quantification. 


\begin{abstract}
A new method using high performance liquid chromatography coupled with electrospray mass spectrometry is described for the quantification of PBMC concentration of tyrosine kinase inhibitors imatinib, dasatinib and nilotinib. A simple PBMC isolation and extraction procedure were applied on 10-14 mL of blood aliquots. Chromatographic separation of drugs and Internal Standard (quinoxaline) was achieved with a gradient (acetonitrile and water + formic acid $0.05 \%$ ) on a $\mathrm{C} 18$ reverse phase analytical column with 25 min of analytical run, at flow rate of $0.25 \mathrm{~mL} / \mathrm{min}$. Mean intra- and inter-day precision for all compounds were 8.76 and $12.20 \%$; mean accuracy was $-3.86 \%$; extraction recovery ranged within 79 and $91 \%$. Calibration curves ranged from 50.0 to $0.25 \mathrm{ng}$. The limit of quantification was set at $0.25 \mathrm{ng}$ for all the analyzed drugs.

This novel developed methodology allows a specific, sensitive and reliable simultaneous intracellular determination of the three tyrosine kinase inhibitors imatinib, dasatinib and nilotinib in a single chromatographic run, useful for drugs estimation in PBMC of patients affected by chronic myeloid leukemia.
\end{abstract}




\section{Introduction}

Chronic myeloid leukemia (CML) is a myeloproliferative disorder, characterized by the presence of the Philadelphia chromosome, consequence of a translocation 9-22, producing a fusion oncogene referred to as BCR-ABL, encoding for a BCR-ABL kinase [1]. Thus, the use of BCR-ABL-targeted therapy is the standard of care for this disease. Current frontline therapy for CML is imatinib (Gleevec $^{\mathrm{TM}}$, STI-571), a 2-phenylaminopyrimidine-type inhibitor of the BCR-ABL kinase [2], that competitively inhibits the binding of ATP to the ATP binding pocket of BCR-ABL [3-4]. In the International Randomized Study of Interferon and STI571 (IRIS; ClinicalTrials.gov number, NCT00006343), imatinib has been associated with a superior response rate and improved progression-free survival, as compared with the previous standard therapy, interferon alfa plus lowdose cytarabine [5-7]. Eight-year follow-up of IRIS revealed that responses to imatinib were durable and had an acceptable adverse-event profile, with an estimated rate of overall survival of $85 \%$ [8]. Although most patients show excellent responses to imatinib treatment, nearly $20 \%$ of patients who take the drug do not have a complete cytogenetic response, and others may have intolerable side effects or drug resistance over time [8]. Resistance, mainly caused by point mutations, leads to a reduced affinity of imatinib for the ATP binding domain of the BCR-ABL protein and to a reactivation of the BCR-ABL kinase activity [9-10]. Loss of response and transformation to advanced disease occur mainly in the first 3 years of imatinib therapy, inducing a rate of overall survival often poor in these patients [8]. To overcome imatinib resistance and intolerance, more potent tyrosine kinase inhibitors (TKIs), such as dasatinib and nilotinib, have been developed for the treatment of CML. Dasatinib (Sprycel ${ }^{\mathrm{TM}}$, BMS-354825) is a structurally distinct drug which has a more potent activity than imatinib [11-12]. It also inhibits Src kinases, proteins that play a critical role in the development, growth, progression, and metastasis of a number of human cancers [13]. Dasatinib offers a new treatment option for patients with CML or Ph-positive acute lymphoblastic leukemia who are either unable to tolerate or resistant to previous therapy, including imatinib. Dasatinib has been found to be more effective in eliciting a cytogenetic 
or hematologic response and better tolerated than high-dose imatinib. However, many factors play a role in determining whether dasatinib may provide benefit to patients. Some of these factors are known, such as BCR-ABL-sensitive mutations, whereas other mechanisms of CML not related to BCR-ABL are unknown or unclear [14]. Another second generation TKI is Nilotinib (Tasigna ${ }^{\mathrm{TM}}$, AMN107). It is a close analog of imatinib with higher potency and selectivity for BCR-ABL kinase inhibition in-vitro and in-vivo than imatinib [15-16]. Nilotinib was first approved in the United States and elsewhere in 2007 for patients with CML in the chronic or accelerated phase who had resistance to or could not tolerate imatinib [17-18]. In a phase 3 study, randomized, open-label, multicenter trial, authors compared the efficacy and safety of nilotinib with that of imatinib. Analysis was done for patients with newly diagnosed Philadelphia chromosome-positive CML in the chronic phase, with the rate of major molecular response at 12 months as the primary end point. Study showed more efficacy for nilotinib [19].

Due to the large TKIs proposal in CML treatment, imatinib-resistant patients may find the right path to reach clinical effectiveness. A good help may be also offered to this purpose by employing of therapeutic drug monitoring (TDM), essential tool, today, for the management of CML patients. Sub-inhibitory drug concentrations and sequential treatment with multiple TKIs can promote, in fact, the selection of BCR-ABL kinase domain mutations in CML patients. Measurement of antileukemia drugs plasma concentrations reached by treated patients, then, can be useful to monitor CML patients over time, leading the evaluation of patient adherence to daily oral therapy, potential drug-drug interactions, treatment efficacy, and severe drug-related adverse events [20-21]. In recent years, numerous laboratories have reported the use of high-throughput bioanalytical procedures for the single and simultaneous quantification of plasma concentrations of antileukemia drugs [22-35]. Based on validated high performance liquid chromatography (HPLC) methods, the plasma pharmacokinetics (PK) of imatinib has been well investigated, while only few paper on the PK of dasatinib and nilotinib are available to date [11-21-36-39]. If data miss about plasmatic PK, no literature exist at all related to intracellular quantification of all TKIs, included imatinib. Topic 
that should be deeply investigated, instead, in order to have a better indication about ongoing treatment efficacy. Measurement of TKIs plasma concentrations, in fact, is a reliable tool to perform TDM; however, only the drugs fraction reaching the intracellular compartment is expected to exert action. Then a convenient correlation should be done, also, between clinical outcome and intracellular drug levels reached in treated patients. As variability in drug PK and inadequate patient compliance, also poor penetration of drugs into body compartments, particularly in leukocytes or peripheral blood mononuclear cell (PBMC), may contribute to the occurrence of sub-therapeutic drug level, leading loss of treatment efficacy. By the way the mechanisms by which TKIs drugs accumulate within cells remain generally unknown and it should be further investigated, because very few data are published to date [26-40-42]. Moreover, a number of transmembrane transport proteins, such as P-glycoprotein, the gene product of $A B C B 1(M D R 1)$, and related ABC (ATP binding cassette) B1 (ABCB1), ABCG2, such as solute carrier 22A1 (SLC22A1), solute carrier organic anion transporter family members 1B1 (SLCO1B1) and SLCO1B3 transporters are known to actively mediate the efflux and uptake of drugs from cells. The genes coding for these transport and regulatory proteins are polymorphic in humans, with consequences on the expression and function, potentially influencing the intracellular levels of TKIs drugs. In particular was observed in CML patients an association of SLCO1B3 polymorphism with intracellular accumulation of imatinib in leukocytes [42]. Thus, as intracellular concentrations of TKIs drugs are influenced by both their physico-chemical properties and host genetic factors, an assay enabling the monitoring of TKIs levels at the site of their pharmacological action, appears to be an essential tool for the ongoing investigations aimed at preventing TKIs therapy failure or toxicity.

Herein, aim of our study was to develop and validate an HPLC method coupled with electrospray mass spectrometry (HPLC-MS) detection for the simultaneous quantification of imatinib, dasatinib and nilotinib in human PBMC.

\section{Experimental}




\subsection{Chemicals}

Imatinib (Glivec ${ }^{\mathrm{TM}}$, STI-571) and nilotinib (Tasigna ${ }^{\mathrm{TM}}$, AMN-107) were kindly supplied by Novartis Pharma AG (Basel, Switzerland); dasatinib (Sprycel ${ }^{\mathrm{TM}}$, BMS-354825) was purchased from Sequoia Research (Pangbourne, United Kingdom). Acetonitrile HPLC grade and methanol HPLC grade were purchased from J.T. Baker (Deventer, Holland). HPLC grade water was produced with MilliDI system coupled with a Synergy 185 system by Millipore (Milan, Italy). Quinoxaline (QX) and formic acid were obtained from Sigma-Aldrich (Milan, Italy). Lymphoprep was purchased from Sentinel Diagnostics (Milan, Italy). Blank cells (PBMCs) were isolated from the blood of healthy donors, kindly supplied by the Blood Bank of the Maria Vittoria Hospital (Turin, Italy). 


\subsection{Stock solutions, standards (STD) and quality controls $(Q C)$}

The stock solutions of imatinib, dasatinib and nilotinib were prepared by dissolving an accurately weighed amount of drug in methanol to obtain a final concentration of $1 \mathrm{mg} / \mathrm{mL}$; all stock solutions were then stored at $-20^{\circ} \mathrm{C}$. The stock solutions were stored maximum 3 months.

The Internal Standars (IS) working solution was prepared with QX [1 $\mu \mathrm{g} / \mathrm{mL}]$ in methanol and HPLC grade water $(50: 50 \mathrm{v} / \mathrm{v})$ and stored at $4^{\circ} \mathrm{C}$ until use. The six calibration standards and three quality controls (QCs) were prepared adding a determined volume of stock solutions, or diluted stock solution, to each blank PBMCs aliquots before storage at $-80^{\circ} \mathrm{C}$, during no more than three months, in a manner similar to that described in other publications [43-44].

Calibration ranges, from STD 6 to STD 1, and QC amount for all drugs are listed in Table 1.

\subsection{PBMC isolation.}

Clinical samples were collected, after obtaining written informed consent according to local Ethics Committee indications, from patients treated with TKIs. Blood samples were collected in two EDTA tubes $(2 \times 7 \mathrm{~mL})$.

PBMCs were isolated from 10 to $14 \mathrm{~mL}$ of blood using lymphoprep density gradient centrifugation (700 $\mathrm{g}, 25 \mathrm{~min}, 4^{\circ} \mathrm{C}$ with a Jouan Centrifuge [Model BR4i, Saint-Herblain, France]) at each sampling, as described previously [44]. PBMCs were then fast washed twice in $40 \mathrm{~mL}$ cold-ice phosphate-buffered saline and centrifuged $\left(750 \mathrm{~g}, 6 \mathrm{~min}, 4^{\circ} \mathrm{C}\right)$.

The resulting pellet of washed PBMCs was dissolved with $1 \mathrm{~mL}$ extraction solution (methanol:water, 70:30 vol/vol), switched in two criovials $\left(500 \mu \mathrm{l}\right.$ each) and then stored at $-80^{\circ} \mathrm{C}$ until analyses, and for no longer than three months.

The time taken to process PBMCs from phlebotomy to methanol extraction solution was less than 1 hour, ensuring that sampling conditions were ice cold to prevent drug loss [45-46]. Blank PBMCs 
isolated from the blood of healthy donors, as previously described, were stored in aliquots of around $5 \times 10^{6}$ cells.

The cell number were afterwards determined by absorbance with a new procedure (paper in progress). This method used 10 microliters of stored unknown number PBMC aliquots versus 10 microliters of known numbers of PBMC reference aliquots. The known number of the reference aliquots were obtained by a Bekman Coulter Z2 (Instrumentation Laboratory, Milan, Italy), and managed by Z2 AccuComp Software (Version 3.01), without the use of the trypan blue exclusion. The cellular volume used to calculate intracellular concentrations of TKIs was 285 femto-Liters, as described previously [47].

\subsection{STD, $Q C$ and samples preparation}

The stored aliquots of STDs, QCs and patient samples PBMCs were defrosted at room temperature. Fifty $\mu \mathrm{l}$ of IS working solution were added to each tube and the samples were vortexed for 10 seconds. STDs, QCs and patient tube samples were sonicated in an ice-water bath three time (Cycle 0,75; Amplitude 80\%), to fully lyse PBMCs, using a sonicator UP-50 H (Dr Hielscher GmbH, Teltow, Germany). After a centrifugation at $\left(7.000 \mathrm{~g}, 10\right.$ minutes at $\left.4^{\circ} \mathrm{C}\right), 10 \mu \mathrm{l}$ of supernatant were used for the cell count, and the remainder supernatant were collected into glass tubes. The remaining pellets were then washed by vortex for 10 seconds with $200 \mu$ of acetonitrile:metanol solution $(50: 50, \mathrm{vol} / \mathrm{vol})$, centrifuged $\left(7.000 \mathrm{~g}, 10\right.$ minutes at $\left.4^{\circ} \mathrm{C}\right)$ and each supernatant was collected in the indicated glass tubes to be treated by vortex-vacuum evaporation to dryness at $60^{\circ} \mathrm{C}$.

Each extract was reconstituted with $60 \mu \mathrm{l}$ of HPLC-grade water and acetonitrile solution (60:40, vol/vol) and $20 \mu \mathrm{l}$ were injected into the column. For validation purposes, all samples were extracted and analyzed in duplicate. All procedure steps were carried out at room temperature, excluding that of sonication. 


\subsection{Chromatographic and MS conditions}

The HPLC-MS instrument used was a Waters system (Milan, Italy), with binary pump model 1525, AF degaser, 717-plus autosampler, and Micromass ZQ mass detector. LC-MS Empower Pro software (version year 2002, Waters; Milan, Italy) was used.

The chromatographic separation was performed at $35^{\circ} \mathrm{C}$ using a column oven, on Atlantis $\mathrm{T} 3 \mathrm{C}-18$ $3 \mu$ column (150x2.1 mm I.D.) (Waters; Milan, Italy), protected by a Security Guard with C18 (4.0x3.0mm I.D.) pre-column (Phenomenex; CA, USA). The chromatographic run was performed with a gradient (Table 2), and the mobile phase was composed by HPLC grade water containing $0.05 \%$ formic acid, for mobile phase A, and HPLC grade acetonitrile containing $0.05 \%$ formic acid, for mobile phase B.

Detector settings were ESI, positive polarity ionization; capillary voltage $3.5 \mathrm{kV}$; source temperature $110^{\circ} \mathrm{C}$; desolvation temperature $350^{\circ} \mathrm{C}$; nitrogen desolvation flow $800 \mathrm{l} / \mathrm{h}$; nitrogen cone flow 100 1/h. Ions detected, in single ion recording (SIR) mode, were $\mathrm{m} / z, 493.8$ with a cone voltage of $45 \mathrm{~V}$ for imatinib, $\mathrm{m} / \mathrm{z} 487.5$ with a cone voltage of $35 \mathrm{~V}$ for dasatinib, $\mathrm{m} / \mathrm{z}, 529.5$ with a cone voltage of 35V for nilotinib and $m / z 313.0$ with a cone voltage of 50V for QX (IS).

\subsection{Specificity and selectivity}

Interference from endogenous compounds was investigated by analysis of six different blank PBMC samples. Moreover interference from some potentially co-administered drugs were evaluated for: zidovudine, didanosine, stavudine, lamivudine, abacavir, tenofovir, emtricitabine, ethambutol, isoniazid, ribavirin, voriconazole, posaconazole and itraconazole (some of many drugs routinely analyzed in our laboratory). An "interfering drug" has been considered as a molecule 
which exhibits a retention time close to $0.3 \mathrm{~min}$ from the analytes, and with the potential capability to cause ion suppression.

\subsection{Matrix effect}

"Matrix effect" was investigated using six different blank PBMC samples and comparing peak areas obtained from standard solutions of a solution of water and acetonitrile (60:40), containing all our analytes at three different concentrations, and peak areas obtained from blanks post-extraction solution with the same amount of analytes, as described by Taylor [48]. Possible "matrix effect" was calculated, as deviation \%, comparing the peak area obtained from the PBMC extract with the peak area obtained from the standard solution.

\subsection{Accuracy, precision, calibration and limit of quantification}

Intra-day and inter-day accuracy and precision were determined by assaying ten spiked PBMC samples at three different concentrations (QCs) for each drug. Accuracy was calculated as the percent deviation from the nominal concentration. Inter-day and intra-day precision were expressed as the standard deviation at each QC concentration. Each calibration curve was obtained using six calibration points in duplicate, and the ranges are listed in Table 1. Calibration curves were created by plotting the peak area ratios of each drugs relative to the IS against the various drugs concentrations in the spiked PBMC standards. A quadratic regression was used for all curves in order to obtain the best fit for all calibration points. The limit of detection (LOD) in PBMC was defined as the concentration that yields a signal-to-noise ratio of $3 / 1$. The lowest concentration levels that could determined with a percent deviation from the nominal concentration and relative standard deviation $<20 \%$, was considered the lowest limit of quantification (LOQ), as requested by international guidelines [49]. 


\subsection{Recovery}

Recovery from PBMC, using the extraction procedures, was assessed by comparing the peak area obtained from multiple analyses $(n=3)$ of spiked samples (QCs) with the peak area from standard solution of all analytes in a solution of water and acetonitrile (60:40) at the same concentrations, as described by international guidelines [49].

\subsection{Stability}

The stability of antileukemia drugs at different conditions has been previously assayed in many articles [3-25-32-34-50]. For this reason stability assays were not performed.

\subsection{Patients PBMC samples}

Patients receiving standard dosing of imatinib, dasatinib or nilotinib, underwent blood sampling after obtaining their informed consent for the measurement of PBMC drugs concentrations. Blood samples were collected in EDTA tube $(2 \times 7 \mathrm{~mL})$ and processed as described above (see section 2.3). 


\section{Results}

Time of analytical run was chosen as $25 \mathrm{~min}$, according to the retention times of substances, their good separation and with the use of a wash column step and its re-equilibration, that allows to reduce potential ghost-peak interferences highlighted without the washing step. Our analyte retention times were $9.6 \pm 0.2$ minutes for imatinib, $10.6 \pm 0.2$ for dasatinib, $12.0 \pm 0.2$ for nilotinib, 14.8 \pm 0.2 for QX. Representative chromatograms of a blank PBMC extracted and STD1 of imatinib, dasatinib and nilotinib are shown in Figure 1A, 1B and 1C, respectively. Representative chromatogram of a imatinib, dasatinib and nilotinib STD6 PBMC extracted and QX is shown in Figure 2. Mean regression coefficient $\left(r^{2}\right)$ of all calibration curves was higher than 0.99 for all analytes.

\subsection{Specificity and selectivity}

The assay did not show any significant interference with other potentially concomitant drugs (see section 2.6). The tested six blank PBMC samples did not show any interference in the retention times analytes windows for each specified ion detected (Figure 1A, 1B and 1C).

\subsection{Accuracy, precision, limit of quantification}

Results of the validation of the method are listed in Table 3 for all analytes. All observed data (intra-day and inter-day precision [R.S.D.\%]) were all below 15.0\%, as request for FDA guidelines [49]. LOQ and LOD are listed in Table 1.

\subsection{Recovery}

Multiple aliquots $(n=3)$ at each of the three QCs amounts were assayed and mean recovery of drugs were $79 \%$ for imatinib, $82 \%$ for nilotinib and $91 \%$ for dasatinib (mean $\mathrm{CV}$, for all drugs, $6.8 \%$ ). Mean IS recovery was $89 \%$ (mean CV 2.0\%). 


\subsection{Matrix effect}

The deviation $\%$ of the peak area at the three amounts for all analytes is comparable, ranging from $13.5 \%$ to $-1.4 \%$ (mean $-6.4 \%$ ), showing the absence of the "matrix effect".

\subsection{Analysis of plasma samples from treated patients}

Method developed was applied for assaying of 52 PBMC samples, corresponding to different points of time-concentration curve, obtained from 40 patients treated with TKI drugs (28 with imatinib, 4 with dasatinib, 8 with nilotinib). Values obtained resulted in the expected range of concentrations, previously defined in our laboratory (unpublished data) and close to published results by different procedure and kind of analyzed cell [26-40-42]. The mean ratios (PBMC/plasma) of Ctrough concentrations were $7.8,35.3$ and 5.7 for imatinib, dasatinib and nilotinib, respectively. 


\section{Discussion and Conclusion}

Targeted therapies using imatinib, dasatinib and nilotinib, based on the inhibition of protein tyrosine kinases, represent currently the therapeutic strategies for treatment of CML. TDM, recently, has become an essential tool for the management of CML patients, particularly for patients taking imatinib [21-39], which efficacy threshold in terms of plasma concentrations is clearly defined. In order to manage primary or acquired resistance to imatinib, clinical studies using dasatinib or nilotinib as second line therapy or combination of therapies with different TKIs, in sequential or simultaneous administration, are currently under evaluation [21-39]. Sub-inhibitory intracellular drug concentrations, probably, and sequential treatment with multiple tyrosine kinase inhibitors promote the selection of BCR-ABL kinase domain mutations in CML patients [51-52]. Poor penetration of drugs into leukocytes or PBMC, inadequate treatment adherence, and variability in drug PK may also contribute to the occurrence of sub-therapeutic drug level. In fact, only the fraction reaching the intracellular compartment is reasonably expected to exert a therapeutic action. Very few data are published to date [26-40-42], probably due to the difficult to develop, validate and use, a reliable method to quantify intracellular drug concentration. In this context, aim of our study, an HPLC-MS method setting for the simultaneous quantification of TKIs in human PBMC, has encountered current need, because no methods for this simultaneous intracellular determination of these drugs, are available up to now.

The method we've developed and validated, based on a very close PBMC isolation and drug extraction procedure published by our group for antiretroviral drugs [44]. This method can be used everywhere, because require instruments available in all laboratories. These procedures were then coupled to HPLC instrument with single mass detector.

For this purpose we have chosen as our internal standard quinoxaline, as it is easy to purchase, inexpensive (compared with deuterated drug isotopes) and reliable in its chemical behavior. This xenobiotic was fully used as IS in our other validated method [25-44-53-60], with UV and mass detectors. Moreover it is not a potential coadministred drug as was the case in other studies [41-42]. 
The method developed is suitable to be used in clinical studies due to its high extraction efficiency, its high sensitivity, its good reproducibility and due to the simultaneous quantification of the three drugs using a small volumes of blood (10-14 mL).

In fact, since no therapeutic window has been clearly defined for the imatinib, dasatinib and nilotinib in PBMC, as in plasma (excluding imatinib [36-38-39]), it could be interesting try to quantify intracellular concentrations of the three drugs and assess at first potential correlation with plasma concentrations, then a concentration-response relationship. At present no reports showed data related to intracellular levels. Moreover, measurement of antileukemia intracellular concentrations, even if more elaborated than TKIs plasma determination, can be useful, as plasma concentration, to evaluate patient adherence to daily oral therapy, treatment efficacy, severe drugrelated adverse events, and potential drug-drug interactions, especially in patients who must take drugs interfering with cytochrome P450 [20].

Moreover, TKIs are substrate of transmembrane transport proteins encoded by ABCB1, SLCO1B1 and SLCO1B3 gene [42]. The genes coding for these transport are polymorphic with consequences on the expression and function, potentially influencing the intracellular levels of TKI drugs.

The LOQ (0.25 ng) and calibration curves included a wide range of imatinib, dasatinib and nilotinib concentrations/amounts, optimized according to expected and founded ranges of drug concentrations in PBMC patients. Choice of limits of these ranges was based on the values available in the clinical reports, too [26-40-42].

Data of the developed assay makes our method suitable, then, to perform imatinib, dasatinib and nilotinib quantification in PBMC CML patients. Reliability of our method has been demonstrated for all drug concentrations; relative error at QCs concentrations, intra-day and inter-day precision (Table 3) indicate the good performances of our method. Absence of interference peaks at the analyte retention times, without a "matrix effect", coupled with an experienced collection procedure and treatment of PBMC [44] allowed accurate measurement of drugs intracellular levels. 


\section{Conclusion}

We report a new method using HPLC-MS for the simultaneous intracellular determination of imatinib, dasatinib and nilotinib in a single chromatographic run. The selectivity of the assay described could be exploited optimally when monitoring all the three substances in PBMC and/or tissues during animal and clinical PK studies of single or concomitant administration. The described HPLC-MS method allows the rapid, simple, sensitive, and selective simultaneous intracellular quantification of the three major antileukemia drugs. This method could be currently used for the clinical study (or monitoring) of PBMC TKIs concentrations in patients treated with imatinib, dasatinib and/or nilotinib. 
References

[1] C.L. Sawyers, Chronic myeloid leukemia. N Engl J Med 340 (1999) 1330-40.

[2] B.J. Druker, M. Talpaz, D.J. Resta, B. Peng, E. Buchdunger, J.M. Ford, N.B. Lydon, H. Kantarjian, R. Capdeville, S. Ohno-Jones, and C.L. Sawyers, Efficacy and safety of a specific inhibitor of the BCR-ABL tyrosine kinase in chronic myeloid leukemia. N Engl J Med 344 (2001) 1031-7.

[3] R. Bakhtiar, L. Khemani, M. Hayes, T. Bedman, and F. Tse, Quantification of the anti-leukemia drug STI571 (Gleevec) and its metabolite (CGP 74588) in monkey plasma using a semiautomated solid phase extraction procedure and liquid chromatography-tandem mass spectrometry. J Pharm Biomed Anal 28 (2002) 1183-94.

[4] B.J. Druker, Imatinib as a paradigm of targeted therapies. Adv Cancer Res 91 (2004) 1-30.

[5] B.J. Druker, F. Guilhot, S.G. O'Brien, I. Gathmann, H. Kantarjian, N. Gattermann, M.W. Deininger, R.T. Silver, J.M. Goldman, R.M. Stone, F. Cervantes, A. Hochhaus, B.L. Powell, J.L. Gabrilove, P. Rousselot, J. Reiffers, J.J. Cornelissen, T. Hughes, H. Agis, T. Fischer, G. Verhoef, J. Shepherd, G. Saglio, A. Gratwohl, J.L. Nielsen, J.P. Radich, B. Simonsson, K. Taylor, M. Baccarani, C. So, L. Letvak, and R.A. Larson, Five-year follow-up of patients receiving imatinib for chronic myeloid leukemia. N Engl J Med 355 (2006) 2408-17.

[6] T.P. Hughes, J. Kaeda, S. Branford, Z. Rudzki, A. Hochhaus, M.L. Hensley, I. Gathmann, A.E. Bolton, I.C. van Hoomissen, J.M. Goldman, and J.P. Radich, Frequency of major molecular responses to imatinib or interferon alfa plus cytarabine in newly diagnosed chronic myeloid leukemia. N Engl J Med 349 (2003) 1423-32.

[7] S.G. O'Brien, F. Guilhot, R.A. Larson, I. Gathmann, M. Baccarani, F. Cervantes, J.J. Cornelissen, T. Fischer, A. Hochhaus, T. Hughes, K. Lechner, J.L. Nielsen, P. Rousselot, J. Reiffers, G. Saglio, J. Shepherd, B. Simonsson, A. Gratwohl, J.M. Goldman, H. Kantarjian, K. Taylor, G. Verhoef, A.E. Bolton, R. Capdeville, and B.J. Druker, Imatinib compared with interferon and low-dose cytarabine for newly diagnosed chronic-phase chronic myeloid leukemia. N Engl J Med 348 (2003) 994-1004.

[8] T.P. Hughes, A. Hochhaus, S. Branford, M.C. Muller, J.S. Kaeda, L. Foroni, B.J. Druker, F. Guilhot, R.A. Larson, S.G. O'Brien, M.S. Rudoltz, M. Mone, E. Wehrle, V. Modur, J.M. Goldman, and J.P. Radich, Long-term prognostic significance of early molecular response to imatinib in newly diagnosed chronic myeloid leukemia: an analysis from the International Randomized Study of Interferon and STI571 (IRIS). Blood 116 (2010) 3758-65.

[9] M.E. Gorre, M. Mohammed, K. Ellwood, N. Hsu, R. Paquette, P.N. Rao, and C.L. Sawyers, Clinical resistance to STI-571 cancer therapy caused by BCR-ABL gene mutation or amplification. Science 293 (2001) 876-80.

[10] N.P. Shah, and C.L. Sawyers, Mechanisms of resistance to STI571 in Philadelphia chromosome-associated leukemias. Oncogene 22 (2003) 7389-95.

[11] A.V. Kamath, J. Wang, F.Y. Lee, and P.H. Marathe, Preclinical pharmacokinetics and in vitro metabolism of dasatinib (BMS-354825): a potent oral multi-targeted kinase inhibitor against SRC and BCR-ABL. Cancer Chemother Pharmacol 61 (2008) 365-76.

[12] L.J. Lombardo, F.Y. Lee, P. Chen, D. Norris, J.C. Barrish, K. Behnia, S. Castaneda, L.A. Cornelius, J. Das, A.M. Doweyko, C. Fairchild, J.T. Hunt, I. Inigo, K. Johnston, A. Kamath, D. Kan, H. Klei, P. Marathe, S. Pang, R. Peterson, S. Pitt, G.L. Schieven, R.J. Schmidt, J. Tokarski, M.L. Wen, J. Wityak, and R.M. Borzilleri, Discovery of N-(2-chloro-6-methylphenyl)-2-(6-(4-(2-hydroxyethyl)- piperazin-1-yl)-2-methylpyrimidin-4- ylamino)thiazole5-carboxamide (BMS-354825), a dual Src/Abl kinase inhibitor with potent antitumor activity in preclinical assays. J Med Chem 47 (2004) 6658-61. 
[13] M.C. Frame, Src in cancer: deregulation and consequences for cell behaviour. Biochim Biophys Acta 1602 (2002) 114-30.

[14] M. Steinberg, Dasatinib: a tyrosine kinase inhibitor for the treatment of chronic myelogenous leukemia and philadelphia chromosome-positive acute lymphoblastic leukemia. Clin Ther 29 (2007) 2289-308.

[15] H. Kantarjian, F. Giles, L. Wunderle, K. Bhalla, S. O'Brien, B. Wassmann, C. Tanaka, P. Manley, P. Rae, W. Mietlowski, K. Bochinski, A. Hochhaus, J.D. Griffin, D. Hoelzer, M. Albitar, M. Dugan, J. Cortes, L. Alland, and O.G. Ottmann, Nilotinib in imatinib-resistant CML and Philadelphia chromosome-positive ALL. N Engl J Med 354 (2006) 2542-51.

[16] E. Weisberg, P.W. Manley, W. Breitenstein, J. Bruggen, S.W. Cowan-Jacob, A. Ray, B. Huntly, D. Fabbro, G. Fendrich, E. Hall-Meyers, A.L. Kung, J. Mestan, G.Q. Daley, L. Callahan, L. Catley, C. Cavazza, M. Azam, D. Neuberg, R.D. Wright, D.G. Gilliland, and J.D. Griffin, Characterization of AMN107, a selective inhibitor of native and mutant BcrAbl. Cancer Cell 7 (2005) 129-41.

[17] M.M. Oken, R.H. Creech, D.C. Tormey, J. Horton, T.E. Davis, E.T. McFadden, and P.P. Carbone, Toxicity and response criteria of the Eastern Cooperative Oncology Group. Am J Clin Oncol 5 (1982) 649-55.

[18] J.E. Sokal, E.B. Cox, M. Baccarani, S. Tura, G.A. Gomez, J.E. Robertson, C.Y. Tso, T.J. Braun, B.D. Clarkson, F. Cervantes, and et al., Prognostic discrimination in "good-risk" chronic granulocytic leukemia. Blood 63 (1984) 789-99.

[19] G. Saglio, D.W. Kim, S. Issaragrisil, P. le Coutre, G. Etienne, C. Lobo, R. Pasquini, R.E. Clark, A. Hochhaus, T.P. Hughes, N. Gallagher, A. Hoenekopp, M. Dong, A. Haque, R.A. Larson, and H.M. Kantarjian, Nilotinib versus imatinib for newly diagnosed chronic myeloid leukemia. N Engl J Med 362 (2010) 2251-9.

[20] M. Baccarani, G. Saglio, J. Goldman, A. Hochhaus, B. Simonsson, F. Appelbaum, J. Apperley, F. Cervantes, J. Cortes, M. Deininger, A. Gratwohl, F. Guilhot, M. Horowitz, T. Hughes, H. Kantarjian, R. Larson, D. Niederwieser, R. Silver, and R. Hehlmann, Evolving concepts in the management of chronic myeloid leukemia: recommendations from an expert panel on behalf of the European LeukemiaNet. Blood 108 (2006) 1809-20.

[21] N. Singh, L. Kumar, R. Meena, and T. Velpandian, Drug monitoring of imatinib levels in patients undergoing therapy for chronic myeloid leukaemia: comparing plasma levels of responders and non-responders. Eur J Clin Pharmacol 65 (2009) 545-9.

[22] A. Awidi, Salem, II, N. Najib, R. Mefleh, and B. Tarawneh, Determination of imatinib plasma levels in patients with chronic myeloid leukemia by high performance liquid chromatography-ultraviolet detection and liquid chromatography-tandem mass spectrometry: methods' comparison. Leuk Res 34 714-7.

[23] S. Bouchet, E. Chauzit, D. Ducint, N. Castaing, M. Canal-Raffin, N. Moore, K. Titier, and M. Molimard, Simultaneous determination of nine tyrosine kinase inhibitors by 96 -well solidphase extraction and ultra performance LC/MS-MS. Clin Chim Acta 412 1060-7.

[24] A. Davies, A.K. Hayes, K. Knight, S.J. Watmough, M. Pirmohamed, and R.E. Clark, Simultaneous determination of nilotinib, imatinib and its main metabolite (CGP-74588) in human plasma by ultra-violet high performance liquid chromatography. Leuk Res 34 702-7.

[25] S. De Francia, A. D'Avolio, F. De Martino, E. Pirro, L. Baietto, M. Siccardi, M. Simiele, S. Racca, G. Saglio, F. Di Carlo, and G. Di Perri, New HPLC-MS method for the simultaneous quantification of the antileukemia drugs imatinib, dasatinib, and nilotinib in human plasma. J Chromatogr B Analyt Technol Biomed Life Sci 877 (2009) 1721-6.

[26] J. Klawitter, Y.L. Zhang, N. Anderson, N.J. Serkova, and U. Christians, Development and validation of a sensitive assay for the quantification of imatinib using LC/LC-MS/MS in human whole blood and cell culture. Biomed Chromatogr 23 (2009) 1251-8. 
[27] K. Micova, D. Friedecky, E. Faber, A. Polynkova, and T. Adam, Flow injection analysis vs. ultra high performance liquid chromatography coupled with tandem mass spectrometry for determination of imatinib in human plasma. Clin Chim Acta 411 1957-62.

[28] M. Miura, N. Takahashi, and K. Sawada, High-performance liquid chromatography with solidphase extraction for the quantitative determination of nilotinib in human plasma. Biomed Chromatogr 24 789-93.

[29] M. Miura, N. Takahashi, and K. Sawada, Quantitative determination of imatinib in human plasma with high-performance liquid chromatography and ultraviolet detection. J Chromatogr Sci 49 412-5.

[30] R.L. Oostendorp, J.H. Beijnen, J.H. Schellens, and O. Tellingen, Determination of imatinib mesylate and its main metabolite (CGP74588) in human plasma and murine specimens by ion-pairing reversed-phase high-performance liquid chromatography. Biomed Chromatogr 21 (2007) 747-54.

[31] R.A. Parise, M.J. Egorin, S.M. Christner, D.D. Shah, W. Zhou, and J.H. Beumer, A highperformance liquid chromatography-mass spectrometry assay for quantitation of the tyrosine kinase inhibitor nilotinib in human plasma and serum. J Chromatogr B Analyt Technol Biomed Life Sci 877 (2009) 1894-900.

[32] S. Pursche, O.G. Ottmann, G. Ehninger, and E. Schleyer, High-performance liquid chromatography method with ultraviolet detection for the quantification of the BCR-ABL inhibitor nilotinib (AMN107) in plasma, urine, culture medium and cell preparations. J Chromatogr B Analyt Technol Biomed Life Sci 852 (2007) 208-16.

[33] O. Roth, O. Spreux-Varoquaux, S. Bouchet, P. Rousselot, S. Castaigne, S. Rigaudeau, V. Raggueneau, P. Therond, P. Devillier, M. Molimard, and B. Maneglier, Imatinib assay by HPLC with photodiode-array UV detection in plasma from patients with chronic myeloid leukemia: Comparison with LC-MS/MS. Clin Chim Acta 411 140-6.

[34] K. Titier, S. Picard, D. Ducint, E. Teilhet, N. Moore, P. Berthaud, F.X. Mahon, and M. Molimard, Quantification of imatinib in human plasma by high-performance liquid chromatography-tandem mass spectrometry. Ther Drug Monit 27 (2005) 634-40.

[35] M. Yuki, Y. Yamakawa, T. Uchida, T. Nambu, T. Kawaguchi, A. Hamada, and H. Saito, Highperformance liquid chromatographic assay for the determination of nilotinib in human plasma. Biol Pharm Bull 34 1126-8.

[36] A. Awidi, A.O. Ayed, N. Bsoul, A. Magablah, R. Mefleh, M. Dweiri, M. Ramahi, E. Arafat, M. Bishtawi, and L. Marie, Relationship of serum imatinib trough level and response in CML patients: long term follow-up. Leuk Res 34 1573-5.

[37] E. Faber, D. Friedecky, K. Micova, M. Divoka, B. Katrincsakova, S. Rozmanova, M. Jarosova, K. Indrak, and T. Adam, Imatinib dose escalation in two patients with chronic myeloid leukemia, with low trough imatinib plasma levels measured at various intervals from the beginning of therapy and with suboptimal treatment response, leads to the achievement of higher plasma levels and major molecular response. Int J Hematol 91 897-902.

[38] B. Peng, M. Hayes, D. Resta, A. Racine-Poon, B.J. Druker, M. Talpaz, C.L. Sawyers, M. Rosamilia, J. Ford, P. Lloyd, and R. Capdeville, Pharmacokinetics and pharmacodynamics of imatinib in a phase I trial with chronic myeloid leukemia patients. J Clin Oncol 22 (2004) 935-42.

[39] S. Picard, K. Titier, G. Etienne, E. Teilhet, D. Ducint, M.A. Bernard, R. Lassalle, G. Marit, J. Reiffers, B. Begaud, N. Moore, M. Molimard, and F.X. Mahon, Trough imatinib plasma levels are associated with both cytogenetic and molecular responses to standard-dose imatinib in chronic myeloid leukemia. Blood 109 (2007) 3496-9.

[40] M. Holdhoff, J.G. Supko, G.L. Gallia, C.L. Hann, D. Bonekamp, X. Ye, B. Cao, A. Olivi, and S.A. Grossman, Intratumoral concentrations of imatinib after oral administration in patients with glioblastoma multiforme. J Neurooncol 97 241-5. 
[41] S. Roche, G. McMahon, M. Clynes, and R. O'Connor, Development of a high-performance liquid chromatographic-mass spectrometric method for the determination of cellular levels of the tyrosine kinase inhibitors lapatinib and dasatinib. J Chromatogr B Analyt Technol Biomed Life Sci 877 (2009) 3982-90.

[42] T. Nambu, A. Hamada, R. Nakashima, M. Yuki, T. Kawaguchi, H. Mitsuya, and H. Saito, Association of SLCO1B3 polymorphism with intracellular accumulation of imatinib in leukocytes in patients with chronic myeloid leukemia. Biol Pharm Bull 34 (2011) 114-9.

[43] S. Colombo, A. Beguin, A. Telenti, J. Biollaz, T. Buclin, B. Rochat, and L.A. Decosterd, Intracellular measurements of anti-HIV drugs indinavir, amprenavir, saquinavir, ritonavir, nelfinavir, lopinavir, atazanavir, efavirenz and nevirapine in peripheral blood mononuclear cells by liquid chromatography coupled to tandem mass spectrometry. J Chromatogr B Analyt Technol Biomed Life Sci 819 (2005) 259-76.

[44] A. D'Avolio, M. Simiele, M. Siccardi, L. Baietto, M. Sciandra, V. Oddone, F.R. Stefani, S. Agati, J. Cusato, S. Bonora, and G. Di Perri, A HPLC-MS method for the simultaneous quantification of fourteen antiretroviral agents in peripheral blood mononuclear cell of HIV infected patients optimized using medium corpuscular volume evaluation. J Pharm Biomed Anal 54 (2011) 779-88.

[45] J. Ford, S.H. Khoo, and D.J. Back, The intracellular pharmacology of antiretroviral protease inhibitors. J Antimicrob Chemother 54 (2004) 982-90.

[46] S.H. Khoo, P.G. Hoggard, I. Williams, E.R. Meaden, P. Newton, E.G. Wilkins, A. Smith, J.F. Tjia, J. Lloyd, K. Jones, N. Beeching, P. Carey, B. Peters, and D.J. Back, Intracellular accumulation of human immunodeficiency virus protease inhibitors. Antimicrob Agents Chemother 46 (2002) 3228-35.

[47] M. Simiele, A. D'Avolio, L. Baietto, M. Siccardi, M. Sciandra, S. Agati, J. Cusato, S. Bonora, and G. Di Perri, Evaluation of the mean corpuscular volume of peripheral blood mononuclear cells of HIV patients by a coulter counter to determine intracellular drug concentrations. Antimicrob Agents Chemother 55 (2011) 2976-8.

[48] P.J. Taylor, Matrix effects: the Achilles heel of quantitative high-performance liquid chromatography-electrospray-tandem mass spectrometry. Clin Biochem 38 (2005) 328-34.

[49] Fda, Guidance for Industry Bioanalytical Method Validation, 2001, pp. Guidance for Industry Bioanalytical Method Validation

Center for Drug Evaluation and Research of the U.S.Department of Health and Human Services Food and Drug Administration.

[50] R. Bakhtiar, J. Lohne, L. Ramos, L. Khemani, M. Hayes, and F. Tse, High-throughput quantification of the anti-leukemia drug STI571 (Gleevec) and its main metabolite (CGP 74588) in human plasma using liquid chromatography-tandem mass spectrometry. J Chromatogr B Analyt Technol Biomed Life Sci 768 (2002) 325-40.

[51] J. Cortes, E. Jabbour, H. Kantarjian, C.C. Yin, J. Shan, S. O'Brien, G. Garcia-Manero, F. Giles, M. Breeden, N. Reeves, W.G. Wierda, and D. Jones, Dynamics of BCR-ABL kinase domain mutations in chronic myeloid leukemia after sequential treatment with multiple tyrosine kinase inhibitors. Blood 110 (2007) 4005-11.

[52] N.P. Shah, B.J. Skaggs, S. Branford, T.P. Hughes, J.M. Nicoll, R.L. Paquette, and C.L. Sawyers, Sequential ABL kinase inhibitor therapy selects for compound drug-resistant BCR-ABL mutations with altered oncogenic potency. J Clin Invest 117 (2007) 2562-9.

[53] L. Baietto, A. D'Avolio, F.G. De Rosa, S. Garazzino, M. Michelazzo, G. Ventimiglia, M. Siccardi, M. Simiele, M. Sciandra, and G. Di Perri, Development and validation of a simultaneous extraction procedure for HPLC-MS quantification of daptomycin, amikacin, gentamicin, and rifampicin in human plasma. Anal Bioanal Chem 396 (2010) 791-8.

[54] L. Baietto, A. D'Avolio, F.G. De Rosa, S. Garazzino, S. Patanella, M. Siccardi, M. Sciandra, and G. Di Perri, Simultaneous quantification of linezolid, rifampicin, levofloxacin, and moxifloxacin in human plasma using high-performance liquid chromatography with UV. Ther Drug Monit 31 (2009) 104-9. 
[55] L. Baietto, A. D'Avolio, G. Ventimiglia, F.G. De Rosa, M. Siccardi, M. Simiele, M. Sciandra, and G. Di Perri, Development, validation, and routine application of a high-performance liquid chromatography method coupled with a single mass detector for quantification of itraconazole, voriconazole, and posaconazole in human plasma. Antimicrob Agents Chemother 54 (2010) 3408-13.

[56] A. D'Avolio, L. Baietto, M. Siccardi, M. Sciandra, M. Simiele, V. Oddone, S. Bonora, and G. Di Perri, An HPLC-PDA method for the simultaneous quantification of the HIV integrase inhibitor raltegravir, the new nonnucleoside reverse transcriptase inhibitor etravirine, and 11 other antiretroviral agents in the plasma of HIV-infected patients. Ther Drug Monit 30 (2008) 662-9.

[57] A. D'Avolio, M. Sciandra, M. Siccardi, L. Baietto, D.G. de Requena, S. Bonora, and G. Di Perri, A simple and sensitive assay for determining plasma tipranavir concentration in the clinical setting by new HPLC method. J Chromatogr B Analyt Technol Biomed Life Sci 848 (2007) 374-8.

[58] A. D'Avolio, M. Siccardi, M. Sciandra, L. Baietto, S. Bonora, L. Trentini, and G. Di Perri, HPLC-MS method for the simultaneous quantification of the new HIV protease inhibitor darunavir, and 11 other antiretroviral agents in plasma of HIV-infected patients. J Chromatogr B Analyt Technol Biomed Life Sci 859 (2007) 234-40.

[59] A. D'Avolio, M. Simiele, L. Baietto, M. Siccardi, M. Sciandra, S. Patanella, S. Bonora, and G. Di Perri, A validated high-performance liquid chromatography-ultraviolet method for quantification of the CCR5 inhibitor maraviroc in plasma of HIV-infected patients. Ther Drug Monit 32 (2010) 86-92.

[60] A. D'Avolio, M. Simiele, M. Siccardi, L. Baietto, M. Sciandra, S. Bonora, and G. Di Perri, HPLC-MS method for the quantification of nine anti-HIV drugs from dry plasma spot on glass filter and their long term stability in different conditions. J Pharm Biomed Anal 52 (2010) 774-80. 

Table 1. Detected mass (Dalton), cone voltage used (Volts) and retention time (RT, in minutes) used to quantify Internal Standard and each drug, and absolute amounts from STD6 to STD1 (LOQ), QCs (QC high, QC medium and QC low) and LOD.

\begin{tabular}{|c|c|c|c|c|c|c|c|c|c|c|c|c|c|}
\hline \multicolumn{4}{|c|}{ Drugs Data } & \multicolumn{10}{|c|}{ Amounts (ng) } \\
\hline Drugs & $\underset{\text { (minutes) }}{\mathbf{R T}}$ & $\begin{array}{c}\text { Mass } \\
\text { (dalton) }\end{array}$ & $\begin{array}{c}\text { Cone } \\
\text { Voltage } \\
\text { (volts) }\end{array}$ & $\begin{array}{c}\text { STD } \\
6\end{array}$ & $\begin{array}{c}\text { STD } \\
5\end{array}$ & $\begin{array}{c}\text { STD } \\
4\end{array}$ & $\begin{array}{c}\text { STD } \\
3\end{array}$ & STD 2 & $\begin{array}{l}\text { LOQ / } \\
\text { STD } 1\end{array}$ & $\begin{array}{c}\text { QC } \\
\text { High }\end{array}$ & $\begin{array}{c}\text { QC } \\
\text { Medium }\end{array}$ & QC Low & LOD \\
\hline Imatinib & 9.6 & 493.80 & 45 & 50 & 10 & 5 & 2 & 0.5 & 0.25 & 8 & 4 & 1 & 0.06 \\
\hline Dasatinib & 10.6 & 487.50 & 35 & 50 & 10 & 5 & 2 & 0.5 & 0.25 & 8 & 4 & 1 & 0.06 \\
\hline Nilotinib & 12.0 & 529.50 & 35 & 50 & 10 & 5 & 2 & 0.5 & 0.25 & 8 & 4 & 1 & 0.06 \\
\hline IS & 14.8 & 313.30 & 50 & - & - & - & - & - & - & - & - & - & - \\
\hline
\end{tabular}


Table 2. Chromatographic condition (gradient): Mobile Phase A (HPLC grade water $+0.05 \%$ formic acid) and Mobile Phase B (HPLC grade acetonitrile $+0.05 \%$ formic acid). The flow was $0.25 \mathrm{ml} / \mathrm{min}$.

\begin{tabular}{|c|c|c|}
\hline TIME (min.) & $\begin{array}{c}\text { \% Mobile Phase } \\
\text { A }\end{array}$ & $\begin{array}{c}\text { \% Mobile Phase } \\
\text { B }\end{array}$ \\
\hline 0.0 & 74 & 26 \\
\hline 0.1 & 73 & 27 \\
\hline 2.0 & 55 & 45 \\
\hline 4.0 & 50 & 50 \\
\hline 9.0 & 40 & 60 \\
\hline 9.5 & 30 & 70 \\
\hline 9.6 & 5 & 95 \\
\hline 14.0 & 5 & 95 \\
\hline 14.1 & 95 & 5 \\
\hline 15.1 & 95 & 5 \\
\hline 25.0 & 75 & 25 \\
\hline
\end{tabular}


Table 3. Accuracy (CV\%), Intraday and Interday Precision, as relative standard deviation (RSD\%), assayed for all drugs ( $\mathrm{n}=10$ ).

\begin{tabular}{|c|c|c|c|c|c|c|c|c|c|}
\hline \multirow[t]{3}{*}{ Drugs } & \multicolumn{3}{|c|}{ QC high } & \multicolumn{3}{|c|}{ QC medium } & \multicolumn{3}{|c|}{ QC low } \\
\hline & \multirow{2}{*}{ Accuracy \% } & \multicolumn{2}{|c|}{ Precision R.S.D. \% } & \multirow{2}{*}{ Accuracy \% } & \multicolumn{2}{|c|}{ Precision R.S.D. \% } & \multirow{2}{*}{ Accuracy \% } & \multicolumn{2}{|c|}{ Precision R.S.D. \% } \\
\hline & & Intra-day & Inter-day & & Intra-day & Inter-day & & Intra-day & Inter-day \\
\hline IMATINIB & $-5.72 \%$ & $7.57 \%$ & $11.04 \%$ & $-4.56 \%$ & $8.54 \%$ & $13.01 \%$ & $-6.63 \%$ & $10.69 \%$ & $14.12 \%$ \\
\hline DASATINIB & $2.68 \%$ & $7.98 \%$ & $10.73 \%$ & $-4.22 \%$ & $8.98 \%$ & $11.28 \%$ & $-3.60 \%$ & $9.53 \%$ & $12.28 \%$ \\
\hline NILOTINIB & $2.05 \%$ & $7.60 \%$ & $10.61 \%$ & $-5.77 \%$ & $7.99 \%$ & $12.56 \%$ & $-8.96 \%$ & $9.94 \%$ & $14.18 \%$ \\
\hline
\end{tabular}


Figure 1. Overlapping of STD1 and blank PBMC extracted ions detection (A, Imatinib; B, Dasatinib; C Nilotinib).
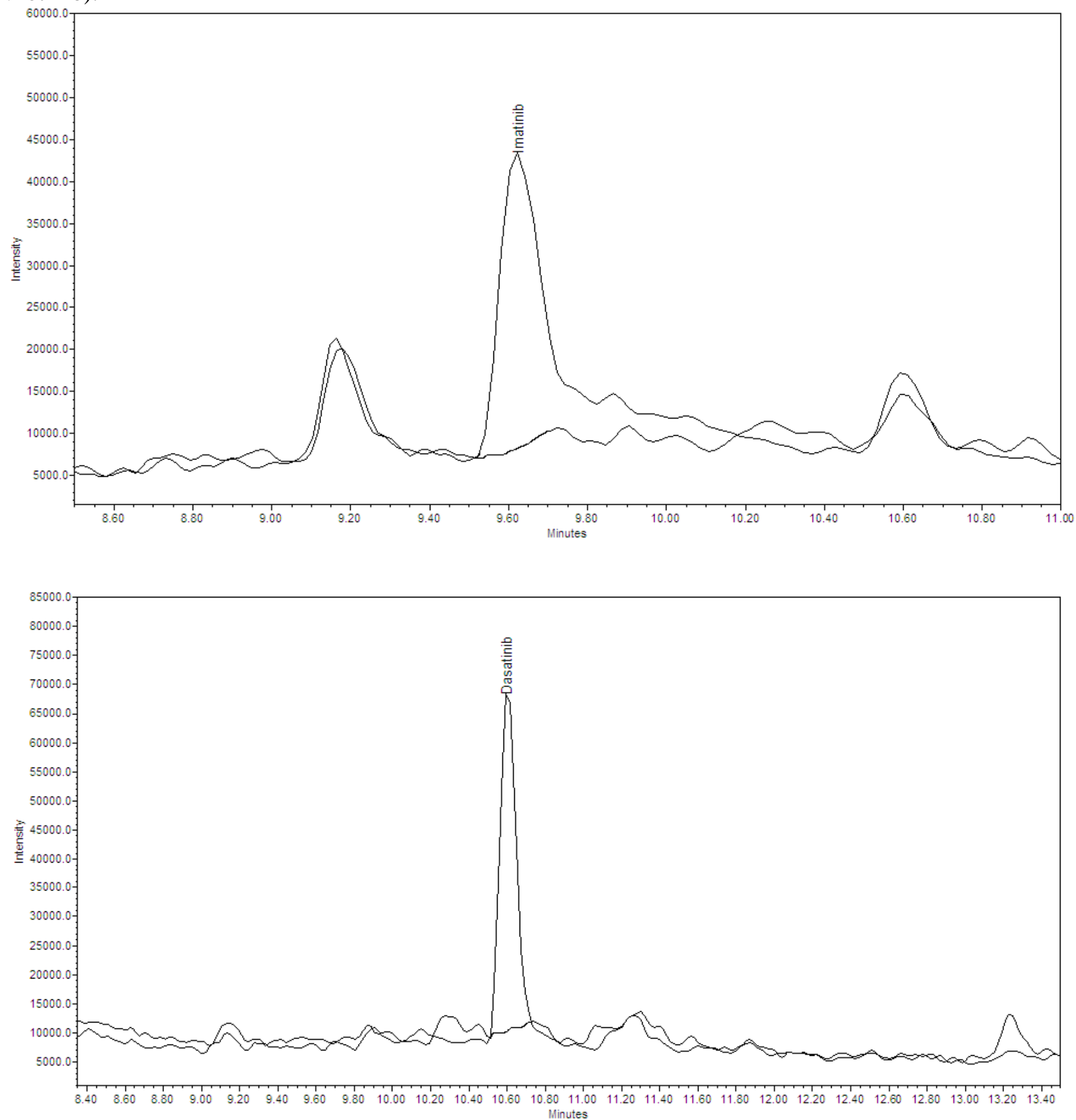


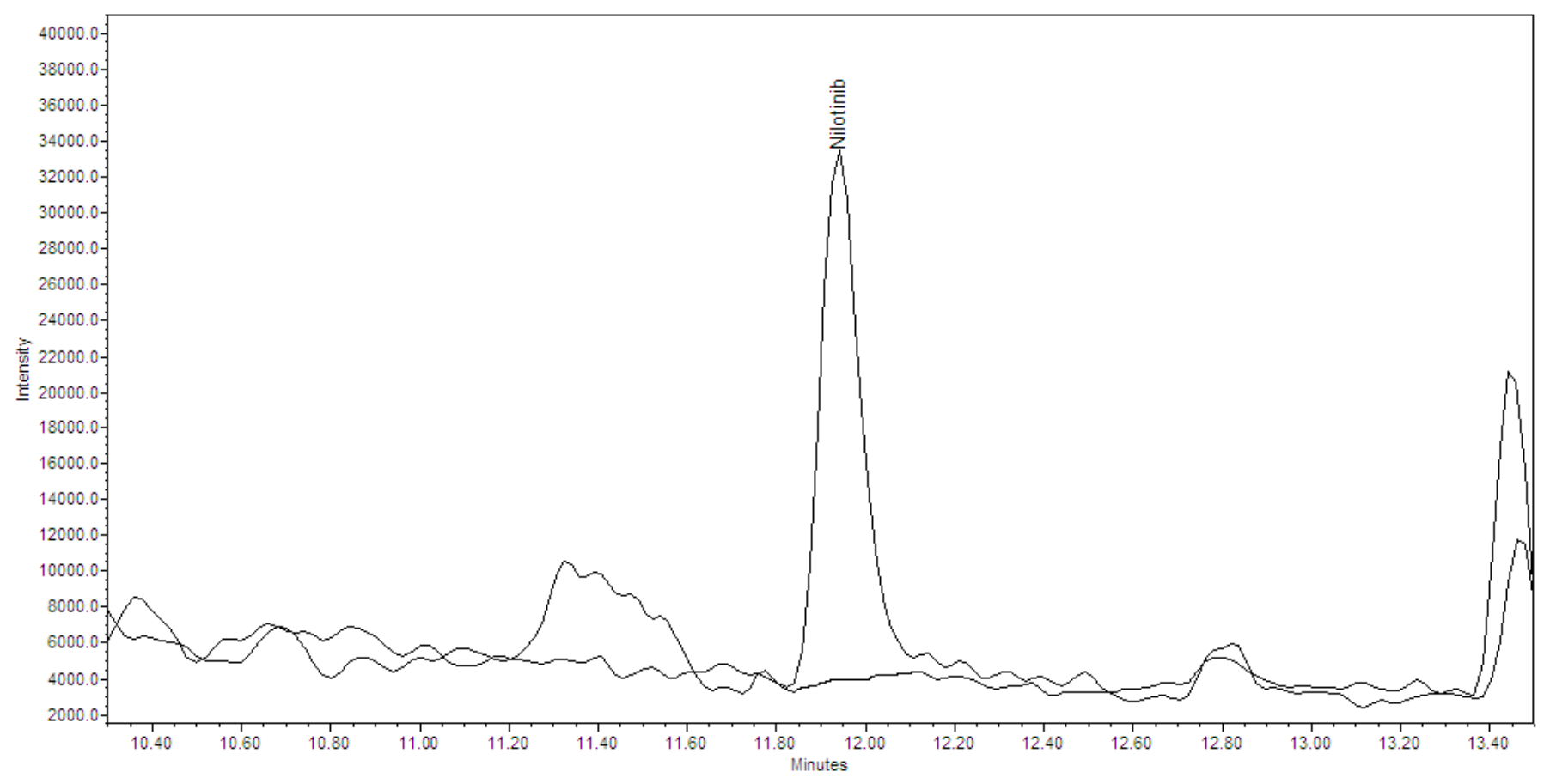


Figure 2. STD6 extracted chromatogram.

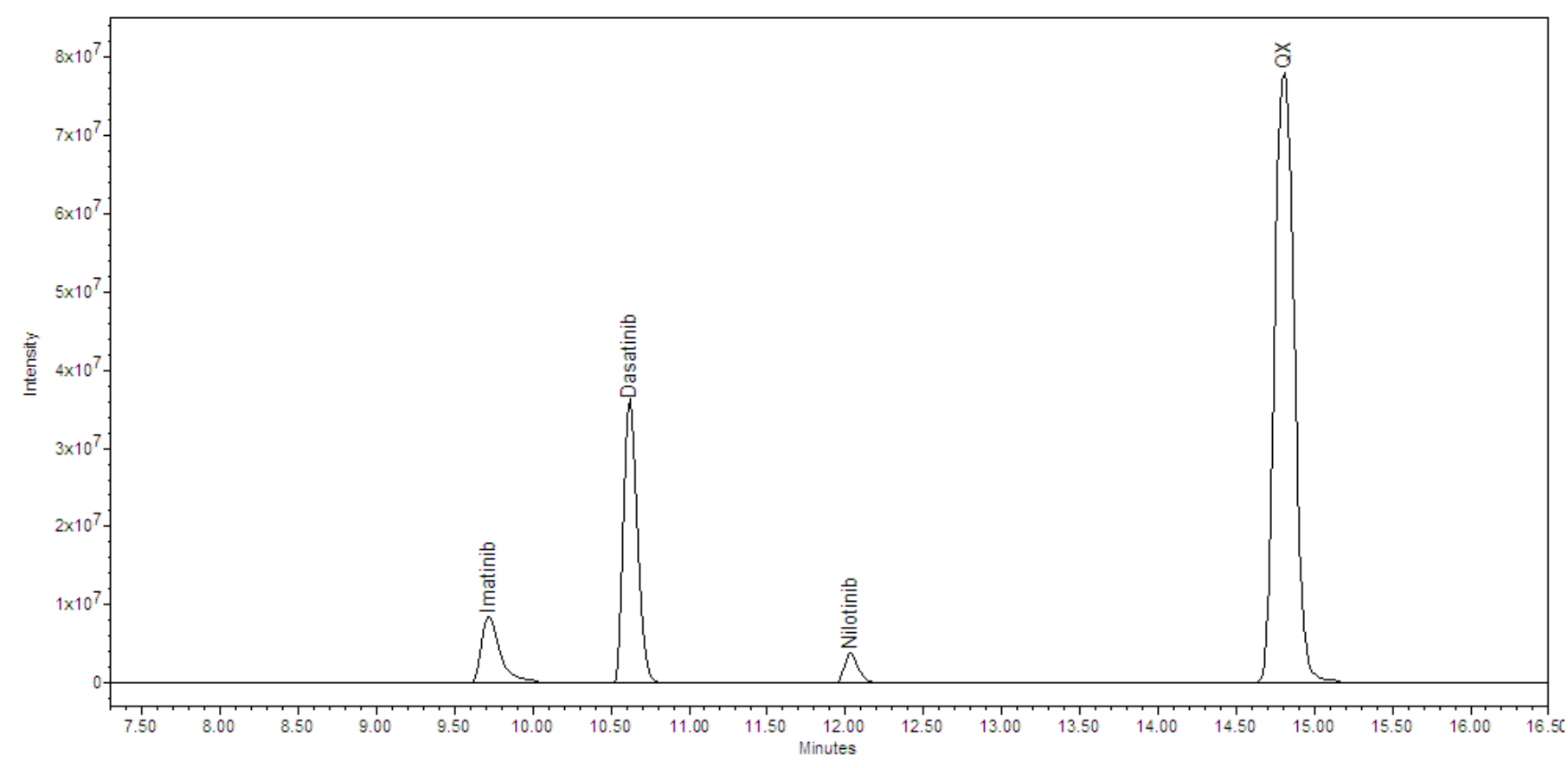

